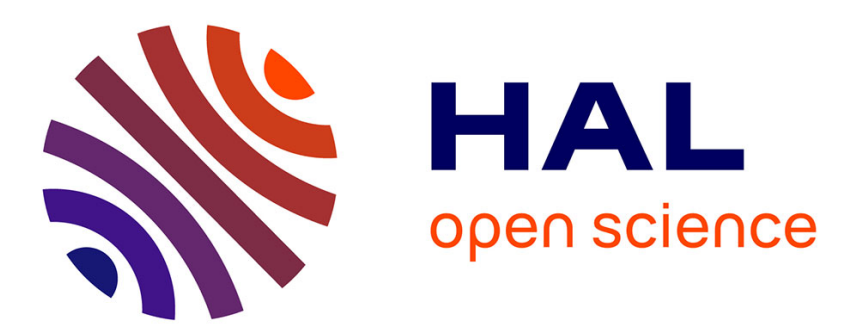

\title{
Fano resonances in photonic crystal slabs near optical bound states in the continuum
}

Cédric Blanchard, Jean-Paul Hugonin, Christophe Sauvan

\section{To cite this version:}

Cédric Blanchard, Jean-Paul Hugonin, Christophe Sauvan. Fano resonances in photonic crystal slabs near optical bound states in the continuum. Physical Review B: Condensed Matter and Materials Physics (1998-2015), 2016, 94 (15), pp.155303 - 155303. 10.1103/PhysRevB.94.155303 . hal01710945

\section{HAL Id: hal-01710945 \\ https://hal-iogs.archives-ouvertes.fr/hal-01710945}

Submitted on 16 Feb 2018

HAL is a multi-disciplinary open access archive for the deposit and dissemination of scientific research documents, whether they are published or not. The documents may come from teaching and research institutions in France or abroad, or from public or private research centers.
L'archive ouverte pluridisciplinaire HAL, est destinée au dépôt et à la diffusion de documents scientifiques de niveau recherche, publiés ou non, émanant des établissements d'enseignement et de recherche français ou étrangers, des laboratoires publics ou privés. 


\title{
Fano resonances in photonic crystal slabs near optical bound states in the continuum
}

\author{
Cédric Blanchard, "Jean-Paul Hugonin, and Christophe Sauvan \\ Laboratoire Charles Fabry, Institut d'Optique Graduate School, CNRS, Université Paris-Saclay, 91127 Palaiseau, France \\ (Received 7 May 2016; revised manuscript received 29 July 2016; published 7 October 2016)
}

\begin{abstract}
Photonic crystal slabs are able to support optical bound states in the continuum. The latter are eigenmodes of the structure that are truly guided (no radiation leakage) despite the fact that they lie above the light cone within the continuum of radiation modes. Such peculiar states can be viewed as modes with an infinite quality factor $Q$. Therefore, the question of the behavior of Fano resonances, as optogeometrical parameters are tuned close to optical bound states in the continuum, is of importance for applications of photonic crystal slabs with ultrahigh $Q$ factors. We study theoretically the reflection and transmission of a photonic crystal slab close to an optical bound state in the continuum with a phenomenological approach involving the poles and zeros of the scattering matrix. In particular, we derive a general relation valid for asymmetric structures that gives the position of a pole in the complex plane as a function of the positions of the zeros. We provide closed-form expressions for the reflection and transmission. The proposed phenomenological approach is validated through rigorous numerical calculations.
\end{abstract}

DOI: 10.1103/PhysRevB.94.155303

\section{INTRODUCTION}

Photonic crystals $(\mathrm{PhCs})$ are artificial structures endowed with a periodic modulation of the refractive index at the wavelength scale [1]. The periodicity of the refractive index leads to the well-known photonic band gap phenomenon. $\mathrm{PhC}$ slabs can be formed by etching a one- or two-dimensional (1D or 2D) $\mathrm{PhC}$ in a dielectric thin layer acting as a waveguide $[2,3]$. Light is then confined in the out-of-plane direction by the refractive index contrast. Compared to three-dimensional (3D) $\mathrm{PhCs}$, the simplified architecture of PhC slabs makes them attractive in the realm of on-chip integrated photonics given that they are vertically compact and benefit from ease of fabrication. These unique features have enabled a wide variety of applications, including antireflection coatings [4], switching devices [5], vertical-cavity surface-emitting laser (VCSEL) [6], gas sensing [7], and structural color generation mechanism [8].

Even if the term photonic crystal is relatively recent, the interest in periodic structures, and more precisely in the way they scatter light, displays a long history. While Lord Rayleigh gave the description of an optical forbidden band as soon as 1887 [9], Wood experimentally discovered in 1902 that weak modifications of the geometrical parameters of a diffraction grating may lead to sharp anomalies in the diffraction spectrum [10]. The so-called Wood's anomalies are now well understood as resulting from the resonant excitation of a leaky mode supported by the PhC slab [11-14]. This optical phenomenon, also known as guided-mode resonance, is a specific case of Fano resonance that has been used to realize resonant filters [15-18]. Around the resonance, the spectral and angular behavior of the reflection and transmission can be accurately described with a phenomenological approach based on the poles and zeros of the scattering matrix $[16,19,20]$. This is the so-called polology framework. For PhC slabs with a weak modulation, this approach provides an in-depth physical understanding since the leaky modes of the corrugated

*Corresponding author: cjfblanchard@gmail.com slab can be understood as perturbations of the guided modes supported by the uncorrugated dielectric slab [17]. But even in the nonperturbative regime of strong modulations, the phenomenological approach is able to accurately describe Fano resonances in PhC slabs. The poles and zeros of the scattering matrix have also been used to describe Fano resonances in metallic gratings [21,22] and in arrays of plasmonic nanoresonators [23].

It was recently pointed out that $\mathrm{PhC}$ slabs can support optical bound states in the continuum (BICs) [24-26]. A BIC is an eigenmode, which is truly guided (no radiation leakage) despite the fact that it lies within the continuum of the radiation modes, i.e., above the light cone in the corresponding dispersion diagram. The existence of such peculiar states strongly depends on the geometrical parameters. In particular, a variation of the slab thickness (all other parameters being fixed) restores the expected leaky character of the mode. Within the polology framework, a BIC corresponds to a real-valued pole of the scattering matrix. Therefore, unlike leaky modes that correspond to complex-valued poles, a BIC can be exactly matched in frequency and wave vector with a propagative plane wave incident on the $\mathrm{PhC}$ slab. Naively, one then expects a divergence of the optical response. However, any divergence of the reflection or transmission is prohibited by energy conservation for a propagative incident wave. Hence, the following basic questions arise. Does the phenomenological model based on the poles and zeros break down for a BIC? What are the values of the reflection and transmission as the parameters are tuned to match those of a BIC? How does a Fano resonance behave as a function of optogeometrical parameters in the vicinity of a BIC?

The aim of this article is to address these intriguing questions. Since a BIC can be viewed as a mode with an infinite quality factor $Q$, the question of Fano resonances close to such peculiar states is of fundamental importance for applications of PhC slabs with ultrahigh $Q$ factors. We will show that the use of the poles and zeros of the scattering matrix is still valid and useful to describe light coupling to a BIC as well as the behavior of the corresponding resonance as geometrical parameters are varied. 
We start Sec. II by a brief reminder of phenomenological expressions for the reflection and transmission coefficients in terms of the poles and zeros of the scattering matrix [19-21]. Then we derive original and general relations between the different parameters (pole, zeros, and prefactors) that appear in the phenomenological expressions. In contrast to previous works [19], these relations are valid for asymmetric structures. In Sec. III we discuss the values of the pole and zeros associated with a BIC. We provide closed-form expressions for the reflection and transmission coefficients. This model allows us to semianalytically describe the evolution of the resonance as a function of any geometrical parameter. Finally, the model is validated in Sec. IV by comparing its predictions with rigorous numerical calculations for three different $\mathrm{PhC}$ slabs: a 1D array of slits in a dielectric membrane, a 2D lattice of circular holes in a dielectric membrane, and a 1D array of dielectric cylinders. Section V concludes the work.

\section{GENERAL PROPERTIES OF THE POLES AND ZEROS IN ASYMMETRIC STRUCTURES}

We consider a dielectric $\mathrm{PhC}$ slab that displays no particular symmetry, as depicted in Fig. 1. The following derivations are completely general aside from two assumptions: (i) there is no absorption in the system and (ii) only one diffraction order is propagative. The latter assumption ensures that the far-field diffraction by the $\mathrm{PhC}$ slab can be fully described by a $2 \times 2$ matrix. The structure can be illuminated by two plane waves with amplitudes $i_{u}$ and $i_{d}$. They give rise to two diffracted plane waves propagating upward (downward) with amplitude $d_{u}\left(d_{d}\right)$, as shown in Fig. 1. The scattering matrix $\mathbf{S}$ is defined from the linear relation connecting the amplitudes of the diffracted waves $\left[d_{u}, d_{d}\right]$ to the incident ones $\left[i_{d}, i_{u}\right]$,

$$
\left[\begin{array}{l}
d_{u} \\
d_{d}
\end{array}\right]=\mathbf{S}\left[\begin{array}{l}
i_{d} \\
i_{u}
\end{array}\right]=\left[\begin{array}{ll}
t_{d} & r_{u} \\
r_{d} & t_{u}
\end{array}\right]\left[\begin{array}{c}
i_{d} \\
i_{u}
\end{array}\right],
$$

where $r_{u}, r_{d}, t_{u}$, and $t_{d}$ are the reflection and transmission coefficients in amplitude. We have chosen this definition of the scattering matrix so that it reduces to the identity matrix II in the absence of any structure.

Since we are considering nonabsorbing materials, energy conservation imposes that $\mathbf{S}$ is unitary and satisfies

$$
\mathbf{S}^{\dagger} \mathbf{S}=\mathbf{S S}^{\dagger}=\mathbb{I},
$$

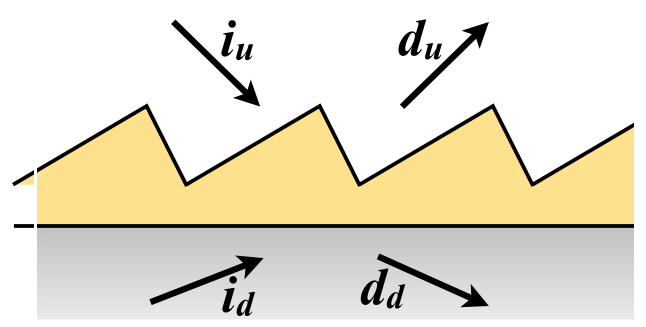

FIG. 1. Generic scheme of an asymmetric PhC slab supporting a single diffraction order. All materials are nonabsorbing. The amplitudes of the incident (diffracted) plane waves are denoted by $i_{u}$ and $i_{d}\left(d_{u}\right.$ and $\left.d_{d}\right)$. The four amplitudes are linearly related through the scattering matrix $\mathbf{S}$ defined in Eq. (1). where the symbol $\dagger$ stands for Hermitian conjugate (conjugate transpose) of the matrix.

In the following we first recall phenomenological expressions for the reflection and transmission coefficients that are valid near a Fano resonance [19-21]. These expressions involve the poles and zeros of the scattering matrix $\mathbf{S}$. Then we derive original and general relations between the different parameters (pole, zeros, prefactors) that appear in the phenomenological expressions.

\section{A. Phenomenological expressions for the reflection and transmission coefficients}

The scattering matrix $\mathbf{S}$ depends on the many parameters of the problem such as the wavelength $\lambda$, the in-plane wave vector, or the geometrical parameters of the PhC slab (thickness $h$, fill factor $F$, period $\Lambda$ ). It also depends on the refractive index $n$ of the material, which may be frequency dependent. The phenomenological approach known as polology consists of using an analytic continuation of $\mathbf{S}$ in the complex plane by considering one of these parameters as a complex variable, all other parameters being fixed. We refer hereafter to this complex variable as $z$. Usually, $z$ represents either the wavelength/frequency or the in-plane wave vector [16,19-21]. Note however that such a choice is not mandatory, $z$ can stand for any other optogeometrical parameter.

Fano resonances in the diffraction spectrum of a $\mathrm{PhC}$ slab are due to the resonant excitation of leaky modes supported by the corrugated slab. Such modes correspond to poles of the scattering matrix extended in the complex plane. All reflection and transmission coefficients are holomorphic functions with the same poles. However, energy conservation imposes them to remain finite when $z$ is taken on the real axis, even in the vicinity of a pole. As a consequence, the coefficients of $\mathbf{S}$ admit a zero close to each pole [19-21]. Accordingly, near a Fano resonance, the reflection and transmission coefficients can be approximated by the following functions:

$$
\begin{aligned}
& r_{u}(z) \approx \dot{r}_{u}(z) \equiv A_{r_{u}} \frac{z-\stackrel{\circ}{z}_{r_{u}}}{z-\hat{z}}, \\
& r_{d}(z) \approx \dot{r}_{d}(z) \equiv A_{r_{d}} \frac{z-\stackrel{\circ}{z}_{r_{d}}}{z-\hat{z}}, \\
& t_{u}(z) \approx \dot{t}_{u}(z) \equiv A_{t_{u}} \frac{z-{\stackrel{\circ}{t_{u}}}_{t_{u}}}{z-\hat{z}}, \\
& t_{d}(z) \approx \dot{t}_{d}(z) \equiv A_{t_{d}} \frac{z-{\stackrel{\circ}{t_{d}}}_{t_{d}}}{z-\hat{z}},
\end{aligned}
$$

where $\hat{z}$ is the pole and $\stackrel{\circ}{z}_{r_{u}}, \stackrel{\circ}{z}_{r_{d}}, \stackrel{\circ}{z}_{t_{u}},{\stackrel{\circ}{t_{d}}}_{t_{d}}$ are the zeros. We use the following notation: $\stackrel{\circ}{r}_{r_{u}}$ is the complex zero of the reflection coefficient from the upper interface of the $\mathrm{PhC}$ slab. These expressions assume that the pole and the zeros are simple and that no other pole or zero exists in the domain of interest. Consequently, the prefactors $A$ are slowly varying functions of $z$. For the sake of simplicity and with no significant loss of accuracy, we assume these functions to be constant. The first structures that have been studied with the polology approach were shallow gratings $[19,21]$. In this case, the prefactors $A$ are usually approximated by the reflection and transmission of the slab without corrugation. But a $\mathrm{PhC}$ is in general more than a simple perturbation of the dielectric slab. Therefore, this 
approximation is not valid and the coefficients $A$ are $a$ priori unknown.

It is noteworthy that the zeros of $\mathbf{S}$ are not independent from one another. It is known that they are related by [19]

$$
\begin{aligned}
& {\stackrel{\circ}{z_{t_{d}}}}^{*}={\stackrel{\circ}{t_{t_{u}}}}, \\
& \stackrel{\circ}{z}_{r_{d}}^{*}={\stackrel{\circ}{z_{r}}}_{r_{u}} .
\end{aligned}
$$

For the sake of completeness, we recall the demonstration of Eq. (4) in Appendix A. We emphasize that these relations hold for any asymmetric structure that satisfies energy conservation and that supports a single diffraction order. Neither reciprocity nor symmetry properties are needed to prove them.

Finally, within the phenomenological framework, the four reflection and transmission spectra near a Fano resonance are fully given by 14 real parameters: four complex coefficients $A$, two complex zeros, and one complex pole. It is well documented in the literature that these parameters are not independent if the structure possesses some symmetry properties. In particular, for a $\mathrm{PhC}$ slab with a mirror symmetry with respect to the horizontal axis, the two zeros are real and a simple relation exists between the complex pole and the real zeros [19]. However, in the general case of a fully asymmetric structure, little is known about the relations between the different parameters. Hereafter, we show that it is not necessary to invoke symmetry properties to derive a few important relations between the parameters in Eq. (3). We provide a general derivation of these relations based on the analytic continuation of the $\mathbf{S}$-matrix unitarity in the complex plane.

Indeed, the unitarity of $\mathbf{S}$ for real values of $z$ given by Eq. (2) can be extended to the complex plane as $[16,19]$

$$
\mathbf{S}^{\dagger}\left(z^{*}\right) \mathbf{S}(z)=\mathbf{S}(z) \mathbf{S}^{\dagger}\left(z^{*}\right)=\mathbb{I},
$$

where the symbol $*$ represents complex conjugate. We recall that if a complex function of a complex variable $f(z)$ is analytic, then $f^{*}\left(z^{*}\right)$ is analytic as well. Equation (5) is equivalent to eight different equations. We will use six of them for the following derivations:

$$
\begin{aligned}
& t_{d}^{*}\left(z^{*}\right) t_{d}(z)+r_{d}^{*}\left(z^{*}\right) r_{d}(z)=1, \\
& r_{u}^{*}\left(z^{*}\right) t_{d}(z)+t_{u}^{*}\left(z^{*}\right) r_{d}(z)=0, \\
& r_{u}^{*}\left(z^{*}\right) r_{u}(z)+t_{u}^{*}\left(z^{*}\right) t_{u}(z)=1, \\
& t_{d}(z) t_{d}^{*}\left(z^{*}\right)+r_{u}(z) r_{u}^{*}\left(z^{*}\right)=1, \\
& t_{d}(z) r_{d}^{*}\left(z^{*}\right)+r_{u}(z) t_{u}^{*}\left(z^{*}\right)=0, \\
& r_{d}(z) r_{d}^{*}\left(z^{*}\right)+t_{u}(z) t_{u}^{*}\left(z^{*}\right)=1 .
\end{aligned}
$$

The derivations of the relations between the parameters in Eq. (3) are based on the following principle. The phenomenological scattering matrix $\dot{\mathbf{S}}(z)$ built with the functions $\dot{r}_{u}(z), \dot{r}_{d}(z), \dot{t}_{u}(z)$, and $\dot{t}_{d}(z)$ is a good approximation of the exact scattering matrix $\mathbf{S}(z)$ for values of $z$ close to the pole $\hat{z}$. Therefore, $\dot{\mathbf{S}}(z)$ satisfies the unitarity relation for $z \approx \hat{z}$. We show that $\dot{\mathbf{S}}(z)$ can in fact be chosen to be unitary for any value of $z$, even if $\dot{\mathbf{S}}(z)$ is not accurate to describe $\mathbf{S}(z)$ as $z$ is moved away from $\hat{z}$. In other words, the functions $\dot{r}_{u}(z), \dot{r}_{d}(z), \dot{t}_{u}(z)$, and $\dot{t}_{d}(z)$ can be chosen to satisfy Eq. (6) for any $z$. This can be done by forcing the coefficients $A_{r_{u}}, A_{r_{d}}, A_{t_{u}}$, and $A_{t_{d}}$ on the one hand and the pole and the zeros on the other hand to satisfy a few important relations, which are given in the next two sections while their demonstrations can be found in the Appendixes.

\section{B. Relations between the coefficients $A$}

By writing Eq. (6) with the phenomenological expressions in Eq. (3) and taking the limit $z \rightarrow \infty$, it is straightforward to show that the coefficients $A_{r_{u}}, A_{r_{d}}, A_{t_{u}}$, and $A_{t_{d}}$ satisfy the following relations:

$$
\begin{aligned}
\left|A_{t_{d}}\right|^{2}+\left|A_{r_{d}}\right|^{2} & =1, \\
\left|A_{r_{u}}\right|^{2} & =\left|A_{r_{d}}\right|^{2}, \\
\left|A_{t_{u}}\right|^{2} & =\left|A_{t_{d}}\right|^{2}, \\
A_{r_{d}} & =-\frac{A_{r_{u}}^{*} A_{t_{d}}}{A_{t_{u}}^{*}} .
\end{aligned}
$$

The details of the derivation can be found in Appendix B. The main consequence of these relations is that the four complex coefficients $A_{r_{u}}, A_{r_{d}}, A_{t_{u}}$, and $A_{t_{d}}$ are not independent. They can be entirely described by four reals parameters $\theta, \varphi_{t_{u}}, \varphi_{r_{u}}$, and $\varphi_{t_{d}}$ that are defined by

$$
\begin{aligned}
A_{t_{u}} & =\cos (\theta) \exp \left[i \varphi_{t_{u}}\right], \\
A_{r_{u}} & =\sin (\theta) \exp \left[i \varphi_{r_{u}}\right], \\
A_{t_{d}} & =\cos (\theta) \exp \left[i \varphi_{t_{d}}\right], \\
A_{r_{d}} & =\sin (\theta) \exp \left[i\left(\varphi_{t_{u}}+\varphi_{t_{d}}-\varphi_{r_{u}}+\pi\right)\right] .
\end{aligned}
$$

The angles $\theta, \varphi_{t_{u}}, \varphi_{r_{u}}$, and $\varphi_{t_{d}}$ are chosen in the interval $[0,2 \pi]$.

\section{Relation between the pole and the zeros}

Furthermore, writing the unitarity of the scattering matrix with the phenomenological expressions leads to a closed-form expression for the pole $\hat{z}$ as a function of the zeros and the parameter $\theta$. We obtain

$$
\begin{aligned}
\hat{z}= & \operatorname{Re}\left({\stackrel{\circ}{z_{u}}}_{t_{u}}\right) \cos ^{2} \theta+\operatorname{Re}\left({\stackrel{\circ}{r_{u}}}\right) \sin ^{2} \theta \\
& +i\left\{\operatorname{Im}^{2}\left({\stackrel{\circ}{t_{u}}}_{t_{u}}\right) \cos ^{2} \theta+\operatorname{Im}^{2}\left({\stackrel{\circ}{r_{u}}}\right) \sin ^{2} \theta\right. \\
& \left.+\sin ^{2} \theta \cos ^{2} \theta\left[\operatorname{Re}\left({\stackrel{\circ}{t_{u}}}\right)-\operatorname{Re}\left({\stackrel{\circ}{r_{u}}}\right)\right]^{2}\right\}^{1 / 2} .
\end{aligned}
$$

The derivation of Eq. (9) can be found in Appendix C. According to Eqs. (8a) and (8b), we have $\cos ^{2} \theta=\left|A_{t_{u}}\right|^{2}$ and $\sin ^{2} \theta=\left|A_{r_{u}}\right|^{2}$. Note that we have chosen the positive square root for the imaginary part of $\hat{z}$. In practice, the sign of the square root that has to be chosen depends on the physical variable represented by $z$. For instance, if one uses the time factor $\exp (-i \omega t), \operatorname{Im}(\hat{z})>0$ if $z$ stands for the wavelength, $z=\lambda$, whereas $\operatorname{Im}(\hat{z})<0$ if $z$ stands for the frequency, $z=\omega$.

Equation (9) gives the position of the pole in the complex plane with respect to the positions of the zeros. We emphasize that, in contrast to previous results, this expression is valid for any asymmetric structure, only energy conservation is required. In the particular case of a $\mathrm{PhC}$ slab symmetric about the horizontal axis, the zeros are real and Eq. (9) reduces to the relation that has been previously derived [19]. 
Equations (8) and (9) show that eight real parameters are sufficient to fully describe the reflection and transmission of a PhC slab near a Fano resonance. These parameters are the angles $\theta, \varphi_{t_{u}}, \varphi_{r_{u}}, \varphi_{t_{d}}$ and the two complex zeros ${\stackrel{\circ}{t_{u}}}_{t_{u}}$ and ${\stackrel{\circ}{r_{u}}}_{r_{u}}$. We will now use this important result to describe the behavior of the resonance as a function of a geometrical parameter in the vicinity of a BIC.

\section{MODEL OF FANO RESONANCES NEAR OPTICAL BOUND STATES IN THE CONTINUUM}

We now employ the phenomenological approach and the general relations derived in Sec. II to address the question of the shape of the Fano resonance associated with a BIC and its evolution as geometrical parameters are varied. We provide in this section closed-form expressions for the reflection and transmission coefficients. This model allows us to semianalytically explore the behavior of the Fano resonance as a function of any geometrical parameter. Hereafter we first discuss the values of the pole, the zeros, the reflection, and the transmission for optogeometrical parameters that exactly match those of a BIC. Then we study the evolution of these quantities as one parameter is varied.

\section{A. Pole and zeros corresponding to a BIC}

Because of the absence of leakage, a BIC corresponds to a real pole of the scattering matrix, $\operatorname{Im}(\hat{z})=0$. A BIC can hence be exactly frequency and wave vector matched with a propagative incident plane wave. This is clearly a very specific and interesting case since neither leaky modes nor usual guided modes can be directly addressed with a propagative wave. Indeed, the first ones correspond to complex poles and the second ones lie, by definition, below the light cone. Since we are considering a nonabsorbing structure, energy has to be conserved for a propagative incident wave. According to Eq. (3), the sole possibility for the reflection and transmission to satisfy energy conservation is to have $\hat{z}=\stackrel{\circ}{z}_{r_{u}}=\stackrel{\circ}{z}_{r_{d}}=\stackrel{\circ}{z}_{t_{u}}=\stackrel{\circ}{z}_{t_{d}}$.

The polology framework provides thus two conditions for the existence of a BIC. First, all zeros have to be real. Second, the zeros have to be equal to the pole $\hat{z}$, which is real by definition of a BIC. As a consequence, the reflection and transmission do not take particular values such as 0 or 1 ; they are simply given by $\left|r_{u}\right|^{2}=\left|A_{r_{u}}\right|^{2},\left|r_{d}\right|^{2}=\left|A_{r_{d}}\right|^{2},\left|t_{u}\right|^{2}=$ $\left|A_{t_{u}}\right|^{2}$, and $\left|t_{d}\right|^{2}=\left|A_{t_{d}}\right|^{2}$. This means that a BIC cannot be excited by an incident plane wave. We emphasize that this situation is drastically different from leaky modes or usual guided modes lying below the light cone that can all be excited by an incident wave, provided that it is evanescent. In this case, the reflection and transmission do not satisfy energy conservation and diverge (nothing unphysical since the incident field is evanescent). We have checked (not shown here) that the field in the $\mathrm{PhC}$ slab in response to an incoming plane wave is totally different from the field profile of the BIC.

\section{B. Evolution of a Fano resonance near a BIC}

$\mathrm{Up}$ to now, BICs have only been observed in $\mathrm{PhC}$ slabs with a mirror symmetry with respect to the horizontal axis [24-26]. We will therefore restrict the following study to such structures. In this case, we have $t_{u}=t_{d}$ and $r_{u}=r_{d}$, which imply that $\stackrel{\circ}{z}_{t_{u}}=\stackrel{\circ}{z}_{t_{d}}$ and $\stackrel{\circ}{z}_{r_{u}}=\stackrel{\circ}{z}_{r_{d}}$. Because of Eq. (4), the zeros of the reflection and transmission coefficient are thus real numbers [19] and the first condition for the existence of a BIC is satisfied. The imaginary part of the pole given by Eq. (9) then reduces to

$$
\operatorname{Im}(\hat{z})=\sin \theta \cos \theta\left[\stackrel{\circ}{z}_{t_{u}}-\stackrel{\circ}{z}_{r_{u}}\right] .
$$

Note that the sign of $\operatorname{Im}(\hat{z})$ is imposed, as mentioned in Sec. II. For the sake of convenience, we write Eq. (10) with a positive sign and let the angle $\theta$ be chosen in the $[0,2 \pi]$ interval in order to obtain the correct sign. The choice of $\theta$ depends on the sign of $\stackrel{\circ}{z}_{t_{u}}-\stackrel{\circ}{z}_{r_{u}}$, which can be positive or negative. Equation (10) confirms that the zeros have to be equal for the pole to be real. In the case of a mirror symmetry with respect to the horizontal axis, Eq. (8) can also be simplified since $\varphi_{t_{d}}=\varphi_{t_{u}}$ and $\varphi_{r_{u}}=\varphi_{t_{u}}+\pi / 2+p \pi$, with $p$ an integer. Finally, the reflection and transmission spectra (as a function of $z$ ) can be fully described by four real parameters, the angles $\theta$ and $\varphi_{t_{u}}$ and the real zeros ${\stackrel{\circ}{z_{u}}}_{t_{u}}$ and $\stackrel{\circ}{z}_{r_{u}}$.

In practice we are not only interested in the reflection and transmission spectra (as a function of the wavelength or the incident angle), but also in the evolution of these spectra as a function of another optogeometrical parameter. We therefore define a real number $u$ that represents this second parameter (different from the one represented by $z$ ). We denote hereafter by $u_{0}$ one particular value of this parameter (corresponding for instance to a BIC) and we look at the evolution of the reflection and transmission spectra (as a function of $z$ ) for values of $u$ close to $u_{0}$.

For this purpose we use a Taylor expansion of the zeros. More precisely, since the imaginary part of the pole is governed by $\stackrel{\circ}{z}_{t_{u}}-\stackrel{\circ}{z}_{r_{u}}$, we expand in Taylor series of $u-u_{0}$ the zero of the transmission $\stackrel{\circ}{z}_{t_{u}}$ and the difference $\stackrel{\circ}{z}_{t_{u}}-\stackrel{\circ}{z}_{r_{u}}$. Since we have assumed that the prefactors $A$ in Eq. (3) are constant with respect to $z$, we also consider them to be constants with respect to the second parameter $u$. The comparisons with exact numerical data in the next section show that this is a reasonable assumption which drastically simplifies the problem [27]. The angles $\theta$ and $\varphi_{t_{u}}$ are thus constant.

For a BIC we have shown that the zeros are real and equal to each other. The zeroth-order coefficient $a_{0}$ in the Taylor expansion of $\stackrel{\circ}{z}_{t_{u}}-\stackrel{\circ}{z}_{r_{u}}$ around a BIC is thus equal to zero. In addition to that, $\operatorname{Im}(\hat{z})$ cannot change its sign as $u$ is varied and the function $\operatorname{Im}(\hat{z})=f(u)$ has to be differentiable for any value of $u$. It follows that the first-order coefficient $a_{1}$ in the Taylor expansion of $\stackrel{\circ}{z}_{t_{u}}-\stackrel{\circ}{z}_{r_{u}}$ has also to be equal to zero [28]. This shows that a BIC corresponds to an extremum equal to zero of the curve ${\stackrel{\circ}{z_{u}}}_{t_{u}}-\stackrel{\circ}{z}_{r_{u}}=f(u)$. For this reason we chose to study the behavior of the resonance as a function of the parameter $u$ is the vicinity of an extremum $u_{0}$ of $\stackrel{\circ}{z}_{t_{u}}-\stackrel{\circ}{z}_{r_{u}}=f(u)$,

$$
\stackrel{\circ}{z}_{t_{u}}-\stackrel{\circ}{z}_{r_{u}}=a_{0}+a_{2}\left(u-u_{0}\right)^{2} .
$$

If $u_{0}$ correspond to a BIC, then we have $a_{0}=0$. Equation (11) evidences that the imaginary part of the pole, and thus the inverse of the $Q$ factor of the Fano resonance, varies quadratically with geometrical parameters. Upon introduction of Eq. (11) into Eq. (9), we get the following expression for the pole:

$$
\hat{z}=\stackrel{\circ}{z}_{t_{u}}+i \sin (\theta)\left[a_{0}+a_{2}\left(u-u_{0}\right)^{2}\right] e^{i \theta} .
$$


Now we introduce the Taylor expansion of $\stackrel{\circ}{z}_{t_{u}}$,

$$
{\stackrel{\circ}{z_{u}}}_{t_{u}}=b_{0}+b_{1}\left(u-u_{0}\right)+b_{2}\left(u-u_{0}\right)^{2} .
$$

Finally, for a PhC slab with a vertical symmetry, the reflection and transmission coefficients in Eq. (3) take the following form as a function of $z$ and $u$ :

$$
\begin{aligned}
& \dot{t}_{u}=\dot{t}_{d}=\cos (\theta) e^{i \varphi_{t u}} \frac{z-b_{0}-b_{1}\left(u-u_{0}\right)-b_{2}\left(u-u_{0}\right)^{2}}{z-b_{0}-b_{1}\left(u-u_{0}\right)-b_{2}\left(u-u_{0}\right)^{2}-i \sin (\theta)\left[a_{0}+a_{2}\left(u-u_{0}\right)^{2}\right] e^{i \theta}}, \\
& \dot{r}_{u}=\dot{r}_{d}=(-1)^{p} i \sin (\theta) e^{i \varphi_{t_{u}}} \frac{z-a_{0}-b_{0}-b_{1}\left(u-u_{0}\right)-\left(a_{2}+b_{2}\right)\left(u-u_{0}\right)^{2}}{z-b_{0}-b_{1}\left(u-u_{0}\right)-b_{2}\left(u-u_{0}\right)^{2}-i \sin (\theta)\left[a_{0}+a_{2}\left(u-u_{0}\right)^{2}\right] e^{i \theta}} .
\end{aligned}
$$

These closed-form expressions allow a semianalytical description of the evolution of a Fano resonance as one optogeometrical parameter is varied. In practice, $z$ is often the wavelength/frequency or the in-plane wave vector and $u$ is a geometrical parameter such as the slab thickness. The parameters $\theta, \varphi_{t_{u}}, a_{0}, a_{2}, b_{0}, b_{1}$, and $b_{2}$ can be fitted from one spectrum for one particular value of $u$. They can then be used to predict reflection and transmission spectra for any other value of $u$ close to $u_{0}$.

Before comparing the model predictions with rigorous numerical calculations, let us discuss the existence of BICs in PhC slabs without mirror symmetry with respect to the horizontal axis. Up to now, BICs in such asymmetric structures have not been demonstrated and therefore their existence can be questioned. Unfortunately, the polology framework does not provide a definitive conclusion; neither the existence nor the nonexistence can be proved. Indeed, no general result exists on the zeros of an asymmetric structure. They are in general complex numbers, but no fundamental reason prevents them to be real. Therefore, the only conclusion that can be drawn from the polology framework is that a BIC could exist in an asymmetric $\mathrm{PhC}$ slab with real zeros.

\section{VALIDATION OF THE MODEL}

In this section the proposed model is validated through comparison with rigorous numerical calculations for three different PhC slabs. We first study light diffraction by a 1D array of slits in a dielectric membrane, see Fig. 2(a). We then calculate a 2D lattice of circular holes in a dielectric membrane, see Fig. 4(a). Finally, we show that the model is also accurate for a 1D array of dielectric cylinders, see Fig. 5(a). Exact numerical calculations for the first two examples are performed with the rigorous coupled-wave analysis (RCWA) [29,30]. For the 2D PhC slab, we have used a specific implementation of the RCWA for diffraction gratings with circular patterns [31]. The 1D array of dielectric cylinders has been calculated with a multipole method adapted to periodic structures $[32,33]$.

\section{A. Lamellar one-dimensional PhC slab}

We consider a lamellar 1D structure, that is a simple array of slits in a dielectric membrane with refractive index $n=3.5$ embedded in air, as shown in Fig. 2(a). The fill factor in dielectric material is $F=0.6$. Figure 2(b) shows the transmission in intensity $T=\left|t_{u}\right|^{2}$ as a function of the wavelength $\lambda$ and the slab thickness $h$, both normalized by the $\mathrm{PhC}$ period $\Lambda$, for an incident angle $\delta=10^{\circ}$. The transmission $T$ has been rigorously calculated with the RCWA. One may observe several particular features for which the transmission experiences a rapid variation from near-unity to near-zero values. They are the signature of Fano resonances corresponding to the resonant excitation of a leaky mode supported by the PhC slab. The resonances seem to disappear for a few points $\left(\lambda_{0}, h_{0}\right)$; some of them are shown by black arrows in Fig. 2(b). These points correspond to BICs: as predicted in Sec. III, the mode is not excited by the incident wave and the transmission does not exhibit any resonance at these peculiar points.

We start by checking the validity of the closed-form expression given in Eq. (14a), which describes the transmission around the resonance. We focus on the first BIC that shows up

(a)
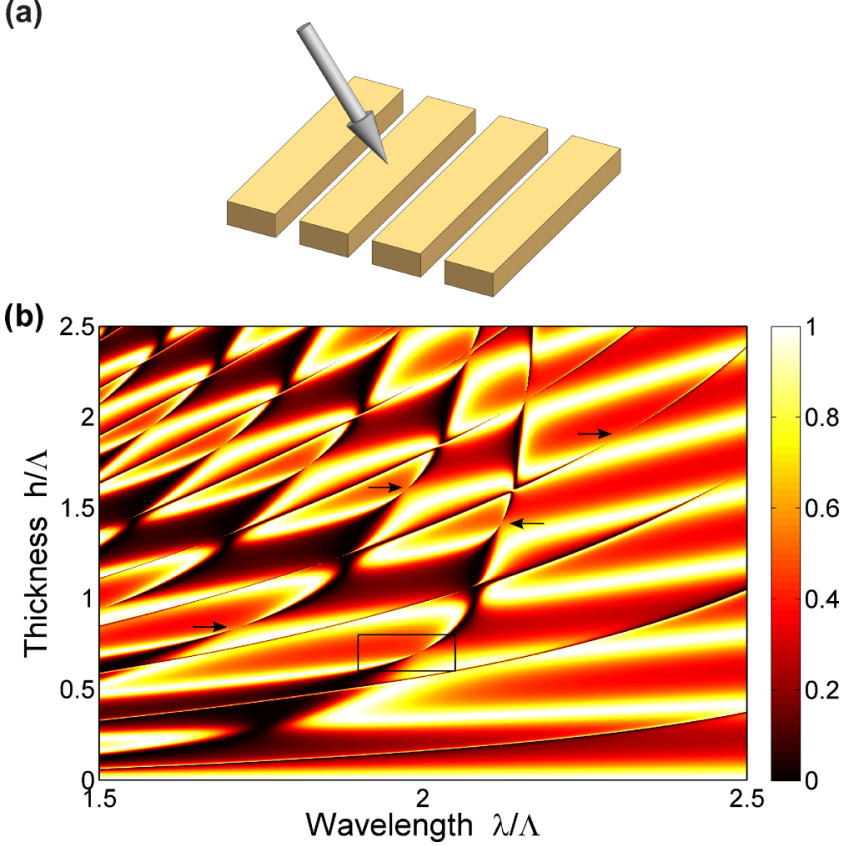

FIG. 2. (a) Lamellar 1D PhC slab in a dielectric membrane $(n=$ $3.5)$ with a thickness $h$. The structure is illuminated by an incident plane wave with an angle $\delta=10^{\circ}$. (b) Transmission in intensity $T=\left|t_{u}\right|^{2}$ as a function of the wavelength $\lambda$ and the thickness $h$ normalized by the period $\Lambda$. The transmission has been rigorously calculated with the RCWA. The arrows show the locations of a few BICs (not all BICs are shown). The rectangle corresponds to the zoomed area that is shown in Fig. 3. 

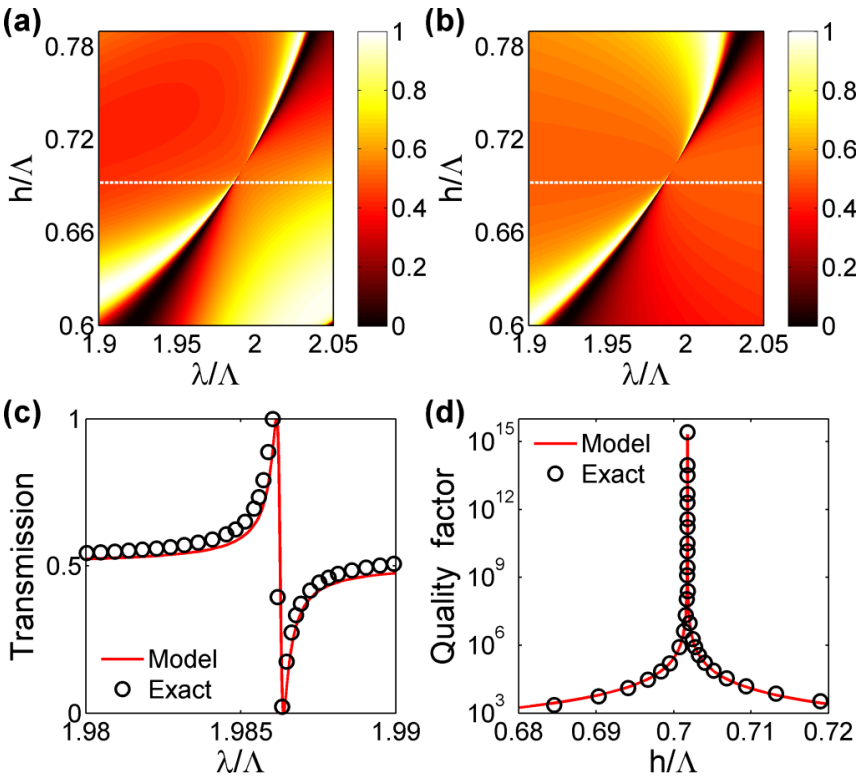

FIG. 3. Lamellar 1D PhC slab. (a) Transmission calculated with the RCWA around the BIC corresponding to the smallest slab thickness (zoom-in of the black rectangle in Fig. 2). (b) Transmission calculated with Eq. (14a) and the fitted parameters. (c) Transmission spectrum near the BIC for $h / \Lambda=0.692$ [see the dashed white lines in (a) and (b)]. (d) Quality factor $Q$ of the Fano resonance as a function of the slab thickness. The point for which $Q$ tends to infinity correspond to the BIC. In (c) and (d) the exact calculation data with the RCWA (black circles) are compared with the predictions of Eq. (14a) (solid red curve).

when the thickness is increased, i.e., at $h_{0} / \Lambda \simeq 0.702$ and $\lambda_{0} / \Lambda \simeq 1.987$. We fit Eq. (14a) to the exact transmission data around this point by taking $z=\lambda / \Lambda$ and $u=h / \Lambda$. The obtained values for the fitting parameters are: $\theta=2.3616 \mathrm{rad}$, $\varphi_{t_{u}}=1.3150 \mathrm{rad}, u_{0}=0.7018, a_{0}=0, a_{2}=-2.3996, b_{0}=$ $1.9923, b_{1}=0.5947$, and $b_{2}=-1.0476$. Figures 3(a) and 3(b) display a zoom-in of the area inside the black rectangle in Fig. 2(b) and offer a comparison between the exact numerical data [Fig. 3(a)] and the model predictions for these fitting parameters [Fig. 3(b)]. The results are in good agreement with each other, which demonstrates the ability of our model to accurately describe the resonance. The discrepancy that can be observed in the bottom right corner is due to the presence of a second resonance nearby, see Fig. 2(b). In order to better visualize the agreement, the transmission spectrum is plotted in Fig. 3(c) for $h / \Lambda=0.692$; this thickness value is highlighted in Figs. 3(a) and 3(b) by white dashed lines. Since we have chosen a thickness value close to a BIC, the curves are reminiscent of a Fano resonance with a high quality factor $Q$.

We now use the model to quantify the variation of the resonance $Q$ factor in terms of the slab thickness $h$. The quality factor can be directly calculated from the complex pole as $Q=\frac{\operatorname{Re}(\hat{z})}{2 \operatorname{Im}(\hat{z})}$. With the model, $Q$ can be analytically predicted, by means of Eq. (12), for any thickness close to $h_{0}$. The results, depicted through the solid red curve in Fig. 3(d), are in excellent agreement with the exact calculation of the complex pole corresponding to the black circles. The $Q$ factor (a)
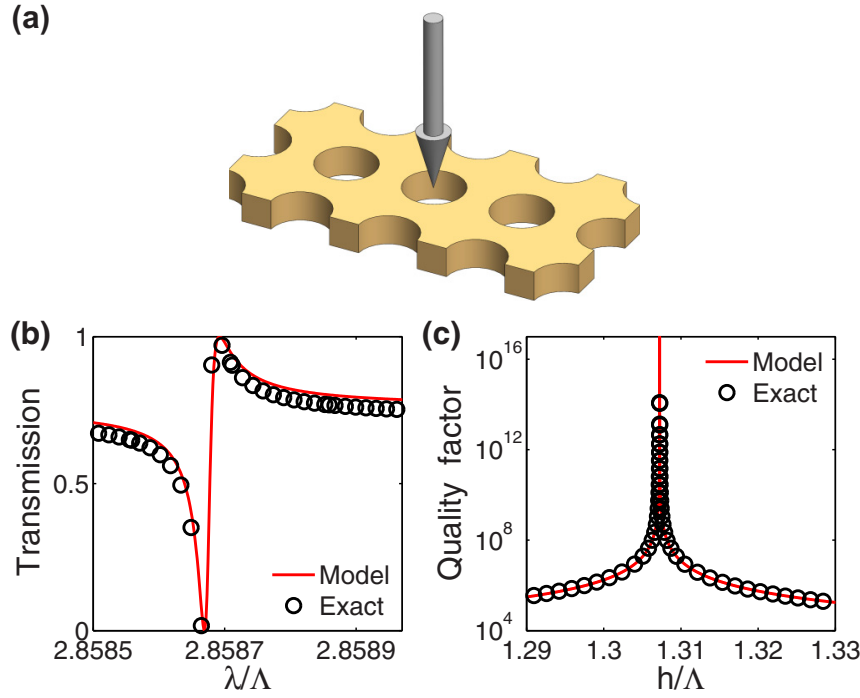

FIG. 4. Two-dimensional PhC slab. (a) A 2D lattice of holes (period $\Lambda$ ) in a dielectric membrane $(n=3.5)$ is illuminated by a plane wave at normal incidence. (b) Transmission spectrum near a BIC for $h / \Lambda=1.282$. (c) Quality factor $Q$ of the Fano resonance as a function of the slab thickness. The point for which $Q$ tends to infinity corresponds to the BIC. In (c) and (d) the exact calculation data with the RCWA (black circles) are compared with the predictions of Eq. (14a) (solid red curve).

is doubtlessly infinite for the BIC at $h_{0} / \Lambda \approx 0.7018$ : we attain $Q>10^{15}$ both with the model and the exact calculation. Such an extremely large value reflects the accuracy of our approach.

\section{B. Two-dimensional PhC slab}

The presented model is naturally valid for $2 \mathrm{D} \mathrm{PhC} \mathrm{slabs.}$ We consider the structure displayed in Fig. 4(a), a square lattice of air holes in a dielectric membrane (refractive index $n=3.5$ ) embedded in air. The lattice period is $\Lambda$ and the hole diameter is $d / \Lambda=0.7$. We recall that previous derivations are valid for a structure that supports only one propagative diffraction order, otherwise the scattering matrix is no longer given by a $2 \times 2$ matrix. We therefore limit this study of 2D structures to normal incidence for avoiding polarization conversion (e.g., a fraction of a TE-polarized incoming wave can be transferred into a TM-polarized diffracted wave) that would result in the existence of two propagative diffraction orders. We use the same methodology as for the 1D lamellar $\mathrm{PhC}$ slab. We determine the parameters in Eq. (14a) by fitting for one thickness close to a BIC the closed-form expression with rigorous calculation data obtained with the RCWA; we get $\theta=0.5150 \mathrm{rad}, \varphi_{t_{u}}=-3.9947 \mathrm{rad}, u_{0}=1.3072, a_{0}=$ $0, a_{2}=0.0366, b_{0}=2.8644, b_{1}=0.2241$, and $b_{2}=-0.2224$. We then use these values to calculate transmission spectra for any thickness and to predict the variation of the $Q$ factor with the slab thickness. In Fig. 4(b) are displayed the transmission spectra for $h / \Lambda=1.282$ calculated with the RCWA and with the model. Figure 4(c) shows the $Q$ factor as a function of $h$ around a BIC. The latter corresponds to the thickness that renders the $Q$ factor infinite. The comparison between 
(a)
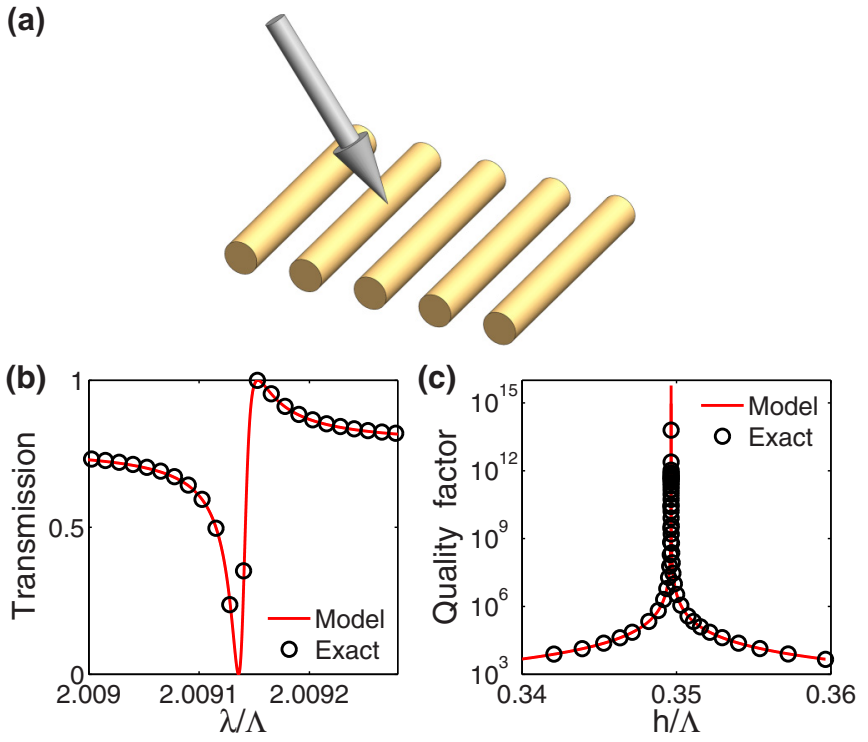

FIG. 5. (a) A 1D array of dielectric cylinders (period $\Lambda$ ) is illuminated by a plane wave with an incident angle $\delta=10^{\circ}$. The diameter of the cylinders is varied and denoted by $h$. (b) Transmission spectrum near a BIC for $h / \Lambda=0.35$. (c) Quality factor $Q$ of the Fano resonance as a function of the slab thickness. The point for which $Q$ tends to infinity correspond to the BIC. In (c) and (d) the exact calculation data with the RCWA (black circles) are compared with the predictions of Eq. (14a) (solid red curve).

rigorous simulations and our model shows again an excellent agreement.

\section{One-dimensional array of dielectric cylinders}

Finally, let us show that the proposed model is also valid to describe Fano resonances associated with BICs in structures more complex than the binary systems previously studied. We consider a 1D array of dielectric cylinders (refractive index $n=3.5$ ) as depicted in Fig. 5(a). The diameter of the cylinders is varied and denoted by $h$. The results can be observed in Figs. 5(b) and 5(c), with again a good agreement between direct numerical calculations and model predictions. In this case we have found the following values for the fitting parameters in Eq. (14a): $\theta=0.4945 \mathrm{rad}$, $\varphi_{t_{u}}=-0.0762 \mathrm{rad}, u_{0}=0.3497, a_{0}=0, a_{2}=5.2820, b_{0}=$ $1.9992, b_{1}=5.4424$, and $b_{2}=-1.0176$.

\section{CONCLUSION}

Photonic crystal slabs are a centerpiece in many practical photonic applications. The understanding of the mechanisms that drive the confinement of light within them is fundamental.
In particular, so-called optical bound states in the continuum (BICs) are intriguing modes that are truly guided (i.e., without radiation leakage), whereas they lie within the continuum of radiation states. In this article we have provided a theoretical analysis of the coupling between incident light and BICs. In particular, we have studied the evolution of the corresponding Fano resonance as optogeometrical parameters are varied. We have used a phenomenological approach based on the study of the poles and zeros of the scattering matrix. We have derived closed-form expressions for the reflection and transmission of the $\mathrm{PhC}$ slab that provide insight into the way light interacts with the system as the parameters are varied. For asymmetric $\mathrm{PhC}$ slabs, we have expressed the position of the pole in the complex plane as a function of the positions of the zeros. The predictions of the phenomenological approach are supported by rigorous numerical calculations for three different cases: a 1D array of slits in a dielectric membrane, a 2D lattice of holes in a dielectric membrane, and a $1 \mathrm{D}$ array of dielectric cylinders.

\section{ACKNOWLEDGMENTS}

The authors acknowledge Philippe Lalanne for fruitful discussions. C.B. acknowledges financial support from Agence Nationale de la Recherche (ANR) on ANR project NEHMESIS.

\section{APPENDIX A: DERIVATION OF THE RELATIONS BETWEEN THE ZEROS}

We recall hereafter the demonstration of Eq. (4), which gives the relations between the zeros of the four reflection and transmission coefficients [19]. This demonstration is based on the analytic continuation of the S-matrix unitarity in the complex plane, see Eqs. (5) and (6).

On the one hand, for $z=\stackrel{\circ}{z}_{t_{d}}\left(z=\stackrel{\circ}{z}_{r_{d}}\right)$, Eq. (6a) simplifies as $r_{d}^{*}\left({\stackrel{\circ}{t_{d}}}^{*}\right) r_{d}\left({\stackrel{\circ}{t_{d}}}_{t_{d}}\right)=1\left[t_{d}^{*}\left({\stackrel{\circ}{r_{d}}}^{*}\right) t_{d}\left({\stackrel{\circ}{r_{d}}}_{r_{d}}\right)=1\right]$, which implies that $r_{d}\left(\stackrel{\circ}{z}_{t_{d}}\right) \neq 0\left[t_{d}\left({\stackrel{\circ}{r_{d}}}_{r^{\prime}}\right) \neq 0\right]$. On the other hand, Eq. (6b) yields

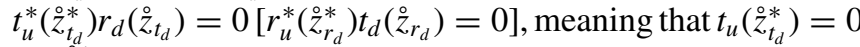
$\left[r_{u}\left(\dot{z}_{r_{d}}^{*}\right)=0\right]$.

This demonstrates that the zeros of the two transmission (reflection) coefficients are complex conjugate of each other, $\stackrel{\circ}{z}_{t d}^{*}=\stackrel{\circ}{z}_{t_{u}}$ and ${\stackrel{\circ}{r_{d}}}_{r_{d}}^{*}=\stackrel{\circ}{z}_{r u}$. It is noteworthy that these relations are true for any structure that can be described by a $2 \times 2$ scattering matrix and that satisfies energy conservation. In particular, reciprocity is not needed.

\section{APPENDIX B: DERIVATION OF THE RELATIONS BETWEEN THE COEFFICIENTS $A_{r_{u}}, A_{r_{d}}, A_{t_{u}}$, AND $A_{t_{d}}$}

In order to derive the relations between the coefficients $A_{r_{u}}, A_{r_{d}}, A_{t_{u}}$, and $A_{t_{d}}$, we introduce the phenomenological expressions given by Eq. (3) in Eq. (6). Then, by multiplying the result by $(z-\hat{z})\left(z-\hat{z}^{*}\right)$, we obtain

$$
\begin{aligned}
& A_{t_{d}}^{*} A_{t_{d}}\left(z-{\stackrel{\circ}{t_{d}}}_{t_{d}}^{*}\right)\left(z-{\stackrel{\circ}{t_{d}}}_{t_{d}}\right)+A_{r_{d}}^{*} A_{r_{d}}\left(z-\stackrel{\circ}{z}_{r_{d}}^{*}\right)\left(z-{\stackrel{\circ}{r_{d}}}_{r^{\prime}}\right)=(z-\hat{z})\left(z-\hat{z}^{*}\right),
\end{aligned}
$$

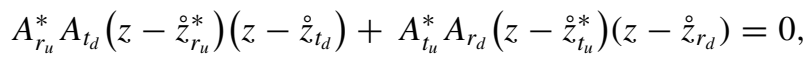

$$
\begin{aligned}
& A_{r_{u}}^{*} A_{r_{u}}\left(z-{\stackrel{\circ}{r_{u}}}^{*}\right)\left(z-{\stackrel{\circ}{r_{u}}}_{r_{u}}\right)+A_{t_{u}}^{*} A_{t_{u}}\left(z-{\stackrel{\circ}{t_{u}}}_{t_{u}}^{*}\right)\left(z-{\stackrel{\circ}{t_{u}}}_{t_{u}}\right)=(z-\hat{z})\left(z-\hat{z}^{*}\right), \\
& A_{t_{d}} A_{t_{d}}^{*}\left(z-{\stackrel{\circ}{t_{d}}}_{t_{d}}\right)\left(z-{\stackrel{\circ}{z_{t}}}_{t_{d}}^{*}\right)+A_{r_{u}} A_{r_{u}}^{*}\left(z-{\stackrel{\circ}{r_{u}}}_{r_{u}}\right)\left(z-{\stackrel{\circ}{r_{u}} *}^{*}\right)=(z-\hat{z})\left(z-\hat{z}^{*}\right),
\end{aligned}
$$




$$
\begin{aligned}
& A_{t_{d}} A_{r_{d}}^{*}\left(z-{\stackrel{\circ}{t_{d}}}_{t_{d}}\right)\left(z-{\stackrel{\circ}{r_{r_{d}}}}^{*}\right)+A_{r_{u}} A_{t_{u}}^{*}\left(z-{\stackrel{\circ}{r_{r_{u}}}}^{\prime}\right)\left(z-{\stackrel{\circ}{t_{t_{u}}^{*}}}^{*}\right)=0, \\
& A_{r_{d}} A_{r_{d}}^{*}\left(z-\stackrel{\circ}{z}_{r_{d}}\right)\left(z-\stackrel{\circ}{z}_{r_{d}}^{*}\right)+A_{t_{u}} A_{t_{u}}^{*}\left(z-\stackrel{\circ}{z}_{t_{u}}\right)\left(z-{\stackrel{\circ}{t_{u}}}^{*}\right)=(z-\hat{z})\left(z-\hat{z}^{*}\right) \text {. }
\end{aligned}
$$

Since we force the phenomenological expressions to satisfy the unitarity relation for any value of $z$, we can take the limit $z \rightarrow \infty$. The system becomes

$$
\begin{aligned}
\left|A_{t_{d}}\right|^{2}+\left|A_{r_{d}}\right|^{2} & =1, \\
A_{r_{u}}^{*} A_{t_{d}}+A_{t_{u}}^{*} A_{r_{d}} & =0, \\
\left|A_{r_{u}}\right|^{2}+\left|A_{t_{u}}\right|^{2} & =1, \\
\left|A_{t_{d}}\right|^{2}+\left|A_{r_{u}}\right|^{2} & =1, \\
A_{t_{d}} A_{r_{d}}^{*}+A_{r_{u}} A_{t_{u}}^{*} & =0, \\
\left|A_{r_{d}}\right|^{2}+\left|A_{t_{u}}\right|^{2} & =1 .
\end{aligned}
$$

These relations lead to Eq. (7) which connects the coefficients $A_{r_{u}}, A_{r_{d}}, A_{t_{u}}$, and $A_{t_{d}}$.

\section{APPENDIX C: DERIVATION OF THE RELATION BETWEEN THE POLE AND THE ZEROS}

We now focus on Eqs. (B1a), (B1c), (B1d), and (B1f). Since $\stackrel{\circ}{z}_{t d}^{*}=\stackrel{\circ}{z}_{t_{u}},{\stackrel{\circ}{r_{d}}}^{*}=\stackrel{\circ}{z}_{r u}$ [see Eq. (4)], $\left|A_{r_{u}}\right|^{2}=\left|A_{r_{d}}\right|^{2}$, and $\left|A_{t_{u}}\right|^{2}=\left|A_{t_{d}}\right|^{2}$ [see Eq. (7)], these four equations are in fact identical,

$$
\begin{aligned}
& \left|A_{t_{u}}\right|^{2}\left(z-\stackrel{\circ}{z}_{t_{u}}\right)\left(z-{\stackrel{\circ}{z_{t u}^{*}}}_{t_{u}}^{*}\right)+\left|A_{r_{u}}\right|^{2}\left(z-\stackrel{\circ}{z}_{r_{u}}\right)\left(z-\stackrel{\circ}{*}_{r_{u}}^{*}\right) \\
& \quad=(z-\hat{z})\left(z-\hat{z}^{*}\right) .
\end{aligned}
$$

For $z=\stackrel{\circ}{z}_{t_{u}}$, this equation becomes

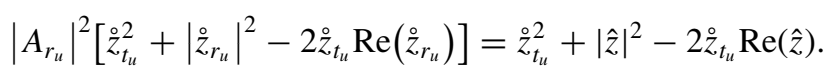

Since $\left|A_{t_{u}}\right|^{2}+\left|A_{r_{u}}\right|^{2}=1$ [see Eq. (7)] we finally get

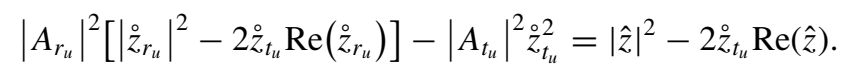

Writing likewise Eq. (C1) for $z={\stackrel{\circ}{r_{u}}}_{r}$ leads to

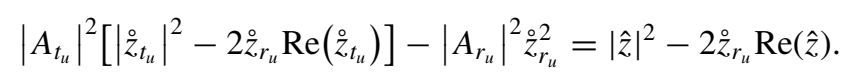

Then, by subtracting Eq. (C4) to Eq. (C3) and using the fact that a complex number $z$ satisfies $|z|^{2}+z^{2}=2 z \operatorname{Re}(z)$, we obtain a closed-form expression for the real part of the pole $\operatorname{Re}(\hat{z})$,

$$
\operatorname{Re}(\hat{z})=\left|A_{r_{u}}\right|^{2} \Re\left({\stackrel{\circ}{r_{u}}}_{u}\right)+\left|A_{t_{u}}\right|^{2} \Re\left({\stackrel{\circ}{t_{u}}}\right) .
$$

Combining this relation with Eq. (C3) yields an expression for the modulus of the pole,

$$
|\hat{z}|^{2}=\left|A_{r_{u}}\right|^{2}\left|{\stackrel{\circ}{r_{u}}}_{u}\right|^{2}+\left|A_{t_{u}}\right|^{2}\left|{\stackrel{o}{t_{u}}}_{t_{u}}\right|^{2} .
$$

Finally, the determination of the imaginary part of the pole is straightforward from Eqs. (C6) and (C5),

$$
\begin{aligned}
& \operatorname{Im}^{2}(\hat{z})=\left|A_{r_{u}}\right|^{2}\left|A_{t_{u}}\right|^{2}\left[\operatorname{Re}\left({\stackrel{\circ}{r_{u}}}\right)-\operatorname{Re}\left({\stackrel{\circ}{z_{u}}}_{u}\right)\right]^{2} \\
& +\left|A_{r_{u}}\right|^{2} \operatorname{Im}^{2}\left({\stackrel{\circ}{z_{u}}}_{r_{u}}\right)+\left|A_{t_{u}}\right|^{2} \operatorname{Im}^{2}\left({\stackrel{\circ}{z_{u}}}_{t_{u}}\right) .
\end{aligned}
$$

Equations (C5) and (C7) lead to the closed-form expression for the complex pole $\hat{z}$ given in Eq. (9).
[1] J. D. Joannopoulos, S. G. Johnson, J. N. Winn, and R. D. Meade, Photonic Crystals: Molding the Flow of Light, 2nd ed. (Princeton University Press, Princeton, 2008).

[2] T. F. Krauss, R. M. De La Rue, and S. Brand, Two-dimensional photonic-bandgap structures operating at near-infrared wavelengths, Nature (London) 383, 699 (1996).

[3] S. G. Johnson, S. Fan, P. R. Villeneuve, J. D. Joannopoulos, and L. A. Kolodziejski, Guided modes in photonic crystal slabs, Phys. Rev. B 60, 5751 (1999).

[4] Y. Kanamori, K. Hane, H. Sai, and H. Yugami, $100 \mathrm{~nm}$ period silicon antireflection structures fabricated using a porous alumina membrane mask, Appl. Phys. Lett. 78, 142 (2001).

[5] X. Letartre, J. Mouette, J. L. Leclercq, P. Rojo-Romeo, C. Seassal, and P. Viktorovitch, Switching devices with spatial and spectral resolution combining photonic crystal and MOEMS structures, J. Lightwave Technol. 21, 1691 (2003).

[6] C. Sciancalepore, B. Ben Bakir, S. Menezo, X. Letartre, D. Bordel, and P. Viktorovitch, III-V-on-Si photonic crystal vertical-cavity surface-emitting laser arrays for wavelength division multiplexing, IEEE Photon. Technol. Lett. 25, 1111 (2013).

[7] C. Blanchard, Q. Lévesque, D. Costantini, C. Jamois, J.-L. Leclercq, A.-L. Coutrot, F. Marquier, L. Milord, C. Grillet, H. Benisty, P. Viktorovitch, X. Letrarte, and J.-J. Greffet, Directional and selective mid-infrared thermal emitters for sensing applications, in Advanced Photonics (Optical Society of America, Boston, 2015), p. SeW2B.2.

[8] Y. Shen, V. Rinnerbauer, I. Wang, V. Stelmakh, J. D. Joannopoulos, and M. Soljacic, Structural colors from Fano resonances, ACS Photon. 2, 27 (2015).

[9] Lord Rayleigh, XVII. on the maintenance of vibrations by forces of double frequency, and on the propagation of waves through a medium endowed with a periodic structure, Philos. Mag. S 5 24, 145 (1887).

[10] R. W. Wood, On a remarkable case of uneven distribution of light in a diffraction grating spectrum, Philos. Mag. 4, 396 (1902). 
[11] U. Fano, The theory of anomalous diffraction gratings and of quasi-stationary waves on metallic surfaces (Sommerfeld's waves), J. Opt. Soc. Am. 31, 213 (1941).

[12] A. Hessel and A. A. Oliner, A new theory of Wood's anomalies on optical gratings, Appl. Opt. 4, 1275 (1965).

[13] M. Neviere, R. Petit, and M. Cadilhac, About the theory of optical grating coupler-waveguide systems, Opt. Commun. 8, 113 (1973).

[14] S. T. Peng, T. Tamir, and H. L. Bertoni, Theory of periodic dielectric waveguides, IEEE Trans. Microwave Theory Tech. 23, 123 (1975).

[15] R. Magnusson and S. S. Wang, New principle for optical filters, Appl. Phys. Lett. 61, 1022 (1992).

[16] A.-L. Fehrembach, D. Maystre, and A. Sentenac, Phenomenological theory of filtering by resonant dielectric gratings, J. Opt. Soc. Am. A 19, 1136 (2002).

[17] A.-L. Fehrembach and A. Sentenac, Study of waveguide grating eigenmodes for unpolarized filtering applications, J. Opt. Soc. Am. A 20, 481 (2003).

[18] Y. Ding and R. Magnusson, Resonant leaky-mode spectral-band engineering and device applications, Opt. Express 12, 5661 (2004).

[19] E. Popov, L. Mashev, and D. Maystre, Theoretical study of the anomalies of coated dielectric gratings, Opt. Acta 33, 607 (1986).

[20] M. Nevière, R. Reinisch, and E. Popov, Electromagnetic resonances in linear and nonlinear optics: Phenomenological study of grating behavior through the poles and zeros of the scattering operator, J. Opt. Soc. Am. A 12, 513 (1995).

[21] M. C. Hutley and D. Maystre, The total absorption of light by a diffraction grating, Opt. Commun. 19, 431 (1976).

[22] S. Collin, G. Vincent, R. Haïdar, N. Bardou, S. Rommeluère, and J.-L. Pelouard, Nearly Perfect Fano Transmission Resonances Through Nanoslits Drilled in a Metallic Membrane, Phys. Rev. Lett. 104, 027401 (2010).

[23] V. Grigoriev, S. Varault, G. Boudarham, B. Stout, J. Wenger, and N. Bonod, Singular analysis of Fano resonances in plasmonic nanostructures, Phys. Rev. A 88, 063805 (2013).
[24] C. W. Hsu, B. Zhen, J. Lee, S.-L. Chua, S. G. Johnson, J. D. Joannopoulos, and M. Soljačić, Observation of trapped light within the radiation continuum, Nature (London) 499, 188 (2013).

[25] B. Zhen, C. W. Hsu, L. Lu, A. D. Stone, and M. Soljačić, Topological Nature of Optical Bound States in the Continuum, Phys. Rev. Lett. 113, 257401 (2014).

[26] C. Blanchard, P. Viktorovitch, and X. Letartre, Perturbation approach for the control of the quality factor in photonic crystal membranes: Application to selective absorbers, Phys. Rev. A 90, 033824 (2014).

[27] We have checked numerically that using Taylor expansions in $u-u_{0}$ of the coefficients $A$ increases the accuracy of the model but at the cost of a loss of simplicity.

[28] If the first-order coefficient $a_{1}$ in the Taylor expansion of $\stackrel{\circ}{z}_{t_{u}}-\stackrel{\circ}{z}_{r_{u}}$ were not equal to zero, the quantity ${\stackrel{\circ}{z_{u}}}_{t_{u}}-\stackrel{\circ}{z}_{r_{u}}$ would change sign around $u_{0}$. Since the sign of $\operatorname{Im}(\hat{z})$ is fixed, $\operatorname{Im}(\hat{z})$ would behave as an absolute value around $u_{0}$ and would not be differentiable.

[29] M. G. Moharam, E. B. Grann, D. A. Pommet, and T. K. Gaylord, Formulations for stable and efficient implementation of the rigorous coupled-wave analysis of binary gratings, J. Opt. Soc. Am. A 12, 1068 (1995).

[30] J.-P. Hugonin and P. Lalanne, Reticolo software, freely available at http://www.lp2n.institutoptique.fr/membresservices/responsables-d-equipe/LALANNE-philippe.

[31] A. David, H. Benisty, and C. Weisbuch, Fast factorization rule and plane-wave expansion method for two-dimensional photonic crystals with arbitrary hole-shape, Phys. Rev. B 73, 075107 (2006).

[32] M. Langlais, J.-P. Hugonin, M. Besbes, and P. Ben-Abdallah, Cooperative electromagnetic interactions between nanoparticles for solar energy harvesting, Opt. Express 22, A577 (2014).

[33] F. Zolla and R. Petit, Method of fictitious sources as applied to the electromagnetic diffraction of a plane wave by a grating in conical diffraction mounts, J. Opt. Soc. Am. A 13, 796 (1996). 